\title{
LOCALLY CATENATIVE SEQUENCES AND TURTLE GRAPHICS*
}

\author{
Juhani KarhumäKI ${ }^{1}$ And Svetlana PUZYninA ${ }^{1,2}$
}

\begin{abstract}
Motivated by striking properties of the well known Fibonacci word we consider pictures which are defined by this word and its variants via so-called turtle graphics. Such a picture can be bounded or unbounded. We characterize when the picture defined by not only the Fibonacci recurrence, but also by a general recurrence formula, is bounded, the characterization being computable.
\end{abstract}

Mathematics Subject Classification. 68R15.

\section{INTRODUCTION}

Combinatorics on words is a relatively new research topic in discrete mathematics, see, e.g., $[1,7,14,15]$. An impressive feature of it is that it is applicable in and motivated by many quite different areas of science, and especially theoretical computer science. In particular, infinite words over a finite alphabet are crucial here. The goal of this paper is to apply the theory and methodology of infinite words to describe two-dimensional pictures. This is done by using infinite words as instructions to turtle graphics.

Turtle graphics is a term in computer graphics for a method of programming vector graphics using a relative cursor upon a Cartesian plane. Papert invented it as a system for translating a sequence of symbols into the motions of an automaton (the "turtle") on a graphics display (see [16]). The basic system is as follows. The turtle has three attributes: a position, an orientation and a pen. We fix a "step

\footnotetext{
Keywords and phrases. Combinatorics on words, locally catenative sequences, turtle graphics, Fibonacci word.

* Supported in part by the Academy of Finland under the grant 121419, by Finnish Cultural Foundation and by Russian Foundation of Basic Research (grants 10-01-00424, 09-01$00244)$.

1 University of Turku, Department of Mathematics, 20014 Turku, Finland

Turku Centre for Computer Science, 20014 Turku, Finland. karhumak@utu.fi

2 Sobolev Institute of Mathematics, Novosibirsk 630090, Russia. svepuz@utu.fi
} 
length" $d$ to be the distance covered by the turtle in one step. We also set $\delta$ to be a given angle, usually $\delta=360^{\circ} / n$ for some integer $n$. The turtle moves with commands that are relative to its own position: "move forward" and "turn left/right by angle $\delta$ ". Turtle graphics is a key feature of the Logo programming language. Turtle geometry is also sometimes used in graphics environments as an alternative to a strictly coordinate-addressed graphics system.

Combined with control flow, procedures, and recursion, the idea of turtle graphics is useful and already used in a Lindenmayer system for giving a geometrical interpretation to the dynamics of $L$-systems and for generating fractals: Koch curve, Dragon curve, Peano curve, Rudin-Shapiro curve, Hilbert curve, etc., see, e.g. $[1,2,8,9,13,17,19]$, and three-dimension generalizations [10]. For a more general treatment of fractal type pictures we refer to [3].

Our starting point is to consider the Fibonacci word, which is known to be one of the most studied infinite words with amazing properties, see [5]. As we see it can define, via turtle graphics, either finite or infinite pictures depending on initial values in its recurrent formulation. This leads to consider all pictures interpreted by a general recurrent formula. As the main result we characterize when such a picture is bounded, the characterization is algorithmic. The characterization is also obtained for recurrence relations with codings over the binary alphabet, which allows to get the result for binary morphic sequences. In the paper we develop new algebraic methods for analyzing such curves.

The paper is organized as follows. After a few preliminary definitions in Section 1, we give in Section 2 examples of curves defined by Fibonacci and ThueMorse words. In Section 3, we develop general theory and establish the necessary and sufficient conditions for boundedness of curves defined by recurrent formulas. Section 4 is devoted to the general recurrences with codings over the binary alphabet. Finally, in Section 5, we give some concluding remarks and discuss open problems.

\section{Preliminaries}

Let $a=\left(a_{n}\right)_{n=0}^{\infty}$ be an infinite word over a finite alphabet $\Sigma$. We translate $a$ to a path visiting integer points of the plane by interpreting letters of $a$ by drawing instructions. Each letter of $\Sigma$ is assigned to a sequence of moves by unit and turns by $90^{\circ}$, which corresponds to a path in the lattice $\mathbb{Z}^{2}$. We start at the origin in the direction $(0,1)$. At step $n$, for $n \geq 0$, the cursor is located at some integer point $\left(x_{n}, y_{n}\right)$ and it has a direction $d_{n}$, which is one of the four directions. Then the cursor draws the path, corresponding to the letter $a_{n}$, in the direction $d_{n}$, starting at $\left(x_{n}, y_{n}\right)$. In the end of this step the cursor comes to an integer point $\left(x_{n+1}, y_{n+1}\right)$ and a direction $d_{n+1}$, determined by the path, corresponding to the letter $a_{n}$.

A locally catenative sequence is a sequence of words in which each word can be constructed as a concatenation of previous words in the sequence [12]. Formally, 
an infinite sequence of words $X_{n}, n \geq 0$, is locally catenative, if for some positive integers $k, i_{1}, \ldots, i_{k}$ and $q=\max \left(i_{1}, \ldots, i_{k}\right)$,

$$
X_{n}=X_{n-1} X_{n-i_{1}} \ldots X_{n-i_{k}}
$$

for all $n \geq q$. We say that $q$ is the order of the corresponding recurrence relation. Some authors use a slightly different definition in which codings of previous words are allowed in the concatenation $[1,20]$. A locally catenative sequence defines the infinite word: $X=\lim _{n \rightarrow \infty} X_{n}$. Actually, the first member in the recurrent relation from the definition of locally catenative sequence is $X_{n-1}$ in order to provide the existence of such limit. It is known, that such word $X$ is a morphic word, see, e.g. $[1,20]$.

A very typical example of a locally catenative sequence is the much studied Fibonacci sequence $F_{n}$, see, e.g., [5], given by

$$
\begin{gathered}
F_{n}=F_{n-1} F_{n-2}, \\
F_{0}=0, F_{1}=01 .
\end{gathered}
$$

The sequence of Thue-Morse words $T_{n}$ is not strictly locally catenative; however, it is locally catenative in the sense of the second definition, because

$$
\begin{aligned}
& T_{n}=T_{n-1} \mu\left(T_{n-1}\right), \\
& T_{0}=0,
\end{aligned}
$$

where the coding $\mu$ replaces 0 with 1 and 1 with 0 .

A celebrated open problem is to decide, whether or not a given morphic word is locally catenative. No general algorithm is known, although the problem was settled in some special cases, for example, for binary alphabets [6]. The intrigue of this problem is supported by the fact that it is just a special case of more general and interesting problem of deciding whether a set of words defined by iterating a morphism, that is a DOL language, is a code, see [4].

The main question we study in this paper is as follows. Suppose we have a locally catenative sequence with initial conditions and drawing instructions. We want to find out whether the corresponding curve is bounded or not.

\section{Curves defined by the Fibonacci and the Thue-Morse SEQUENCES}

In this section we study some simple examples of the pictures corresponding to the Fibonacci and the Thue-Morse words; general theory is developed in the next sections. The Fibonacci word $w$ is the fixed point of the morphism $\varphi(0)=01$, $\varphi(1)=0$. This word is a Sturmian word, so the numbers of 1's in two different subwords of length $n$ differ at most by 1 , see [14]. As it was already mentioned in 
the previous section, this word can also be represented as the limit of the sequence $F_{n}$ given by (1.1).

In this section we consider the following drawing rules. We start at the origin in direction $(0,1)$. At step $n$, for $n \geq 1$, we move straight ahead one unit in the direction we are already moving, if $w_{n}=0$, and turn left by $90^{\circ}$ and move one unit, if $w_{n}=1$. Remark that the question about the boundedness of picture defined by the Fibonacci sequence via these drawing rules has been asked by Saari [18]; actually, answering this question was a starting point of our research. In the further sections we consider general drawing rules and catenative sequences.

In this section we will describe completely the curve corresponding to $F_{n}$ and we will prove that:

- the curve corresponding to the Fibonacci word visits every integer point in $(-\infty, 0] \times[1,+\infty)$;

- the curve expands very slowly: in every 6 iterations the side of the square where the curve stays increases only by 2 .

In Figure 1 one can see the curves corresponding to the iterations $F_{i}$ of the Fibonacci sequence. On each picture on the Cartesian plane the curve is indicated by a bold line, an arrow corresponds to the configuration in the end of iteration, i.e., the position of the point and the direction. The curves on every sixth iteration are similar to each other; the first eleven curves look a little bit different. The proof is a straightforward inductive argument. The base of induction consists of 17 substeps, where first 11 iterations are special, and the next 6 iterations are the same as in general. The inductive step consists of six iterations (substeps). Each substep is done straightforwardly by joining the picture from the previous step and rotated and translated picture from the step before the previous step.

Observe that in the case of the Fibonacci recurrence the length of the sequence $F_{n}$ grows exponentially, but the curve grows only polynomially (actually, linearly).

Now we proceed to the picture corresponding to the Thue-Morse word, which is a fixed point of the morphism $\varphi(0)=01, \varphi(1)=10$ [14]. As it was already mentioned in the previous section, this word can also be represented as the limit of the sequence $T_{n}$ given by (1.2). We consider the same drawing instructions as for the Fibonacci sequence.

In the case of the Thue-Morse sequence the curve is bounded, the corresponding picture is in Figure 2a. The proof is as follows. One can easily draw the picture corresponding to the curve $T_{4}$ of length 16 (see Fig. 2b). In the end of the word $T_{4}$ we come to the initial point $(0,0)$ and have the same direction as the one we started with. One can easily draw the picture corresponding to the curve $t_{17} \ldots t_{32}=\left(T_{4}\right)^{-1} T_{5}$ (see Fig. $2 \mathrm{c}$ ). In the end of the word $T_{5}$ we again come to the initial point $(0,0)$ and have the same direction as the one we started with. The defintion (1.2) implies that if we split the Thue-Morse word to the subwords of length 16 , each of them will be either $T_{4}$ or $\left(T_{4}\right)^{-1} T_{5}$. Hence the whole curve is just the union of these two curves. 

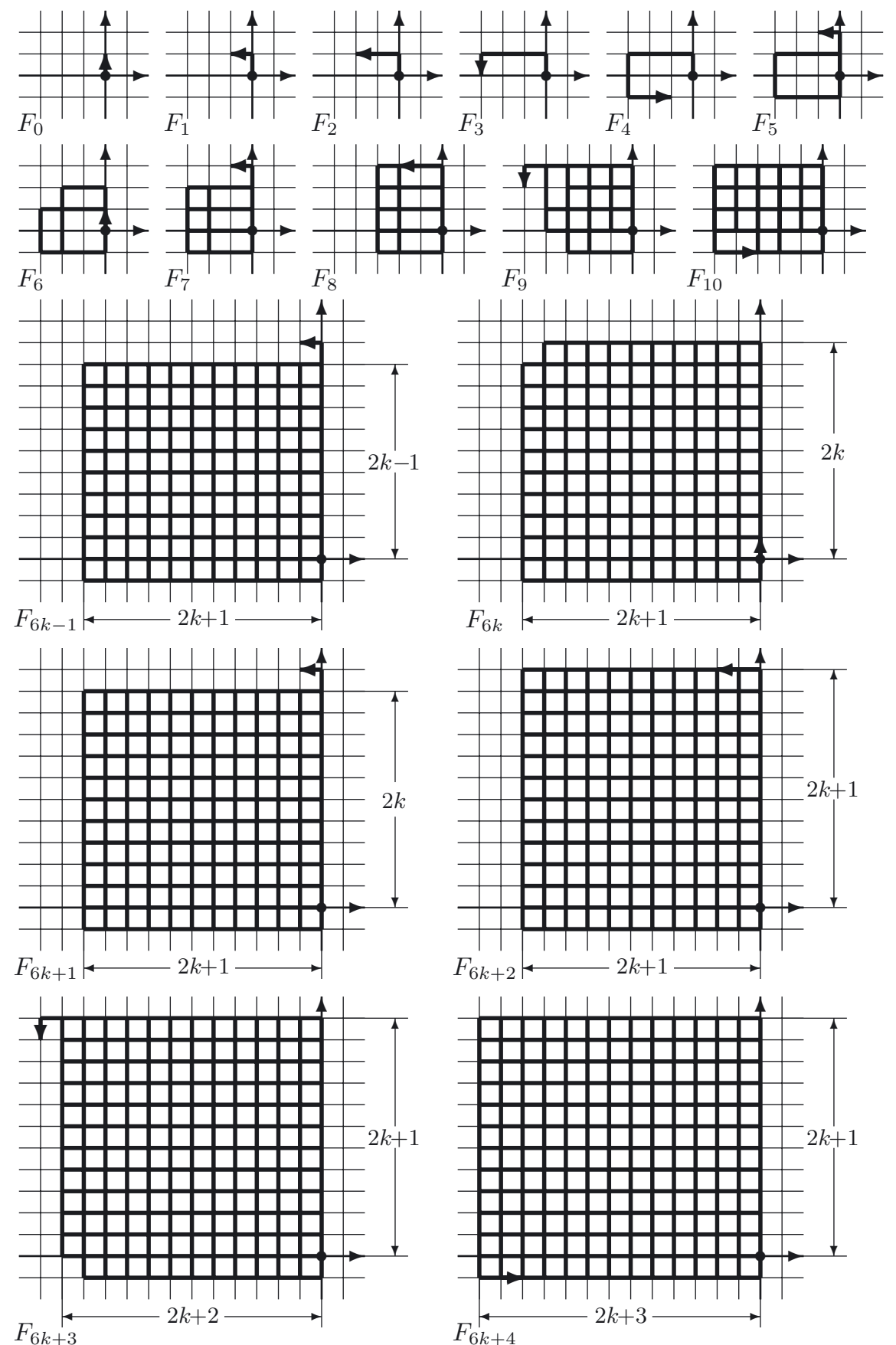

Figure 1. Curves corresponding to iterations of the Fibonacci sequence, $k \geq 2$. 

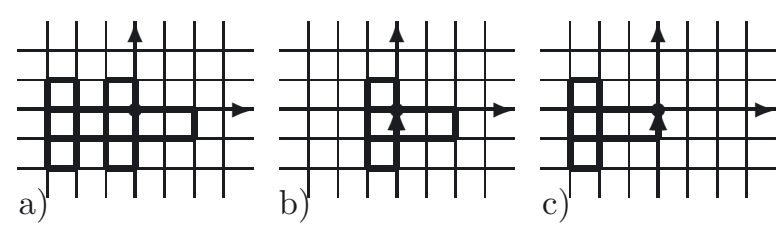

Figure 2. (a) The curve corresponding to the Thue-Morse word. (b) The curve corresponding to the word $T_{4}$. (c) The curve corresponding to the word $\left(T_{4}\right)^{-1} T_{5}$.

It is not difficult to construct drawing rules and/or initial values to obtain a bounded curve corresponding to the Fibonacci recurrence, as well as unbounded curve for the Thue-Morse recurrence. For example, for the initial values $F_{0}=01$, $F_{1}=01011111$ the curve defined by the Fibonacci recurrence is bounded. Equivalently, to obtain bounded curve defined by the Fibonacci recurrence we can keep the initial values $F_{0}=0, F_{1}=01$ and change the drawing instructions as follows: at step $n$, if $w_{n}=0$, move straight ahead one unit, then turn left by $90^{\circ}$ and move one unit; if $w_{n}=1$, move straight ahead one unit, then repeat five times turn left by $90^{\circ}$ and move one unit. The curve defined by the Thue-Morse recurrence with the initial value $T_{0}=0000$ is unbounded. Equivalently, unbounded curve defined by the Thue-Morse recurrence can be obtained using standart initial value $T_{0}=0$, and changing the drawing instructions as follows: at step $n$, if $w_{n}=0$, move straight ahead four units; if $w_{n}=1$, repeat four times turn left by $90^{\circ}$ and move one unit.

To conclude this section, we remark that boundedness of figures defined by general catenative sequences is not a trivial property.

\section{Boundedness of CURVES DEFined By CATENATIVE SEQUENCES}

In this section we develop general theory for necessary and sufficient conditions for boundedness of curves defined by locally catenative sequences.

Let $X_{n}$ be a locally catenative sequence over a finite alphabet $\Sigma$ with the recurrence relation

$$
X_{n}=X_{n-1} X_{n-i_{1}} \ldots X_{n-i_{k}}
$$

and the initial values

$$
X_{0}, \ldots, X_{q-1} \text {. }
$$

Recall that this sequence defines the unique infinite word $X=\lim _{n \rightarrow \infty} X_{n}$.

The letters of $\Sigma$ are associated with drawing instructions, i.e., each letter of $\Sigma$ is assigned to a sequence of moves by unit and turns by $90^{\circ}$. We translate a word $X$ to a path in the lattice $\mathbb{Z}^{2}$ using these rules as instructions to turtle graphics. 
For the characterization of boundedness we will need some notation. Namely, we define the sequence and the group of configurations for recurrence sequence. These definitions are crucial for our considerations.

A configuration $(x, y, d)$ is an integer point $(x, y)$ and a direction $d=0,1,2$ or 3 , corresponding to the directions $(0,1)$ ("up"), (-1,0) ("left"), $(0,-1)$ ("down") and $(1,0)$ ("right"), respectively. Actually the set of configurations is $\mathbb{Z}^{2} \times \mathbb{Z}_{4}$. For a configuration $\beta=(x, y, d)$ denote by $\left.\beta\right|_{\mathbb{Z}^{2}}$ corresponding point $(x, y)$ without direction $d:\left.\beta\right|_{\mathbb{Z}^{2}}=(x, y)$.

We translate a word $Z \in \Sigma^{*}$ to a curve in the following way. We start in the configuration $(0,0,0)$. At step $n$, for $n \geq 0$, we are at some configuration $\left(x_{n}, y_{n}, d_{n}\right)$, so we draw a path, corresponding to the $n$-th letter $z_{n}$ of $Z$, in the direction $d_{n}$, starting at $\left(x_{n}, y_{n}\right)$. In the end of this step we come to a configuration $\left(x_{n+1}, y_{n+1}, d_{n+1}\right)$, determined by the drawing instructions for the letter $z_{n}$. In the further text, by $Z$-curve we mean the curve corresponding to the word $Z$, and the final configuration to which we come after drawing $Z$-curve will be denoted by $\operatorname{conf}(Z)$.

If finite words $Y$ and $Z$ correspond to configurations $(x, y, d)$ and $\left(x^{\prime}, y^{\prime}, d^{\prime}\right)$, then it is not difficult to find a configuration $\left(x^{\prime \prime}, y^{\prime \prime}, d^{\prime \prime}\right)$ corresponding to the word $Y Z$ :

$$
\left(x^{\prime \prime}, y^{\prime \prime}, d^{\prime \prime}\right)= \begin{cases}\left(x+x^{\prime}, y+y^{\prime}, d+d^{\prime}(\bmod 4)\right), & \text { if } d=0, \\ \left(x-y^{\prime}, y+x^{\prime}, d+d^{\prime}(\bmod 4)\right), & \text { if } d=1, \\ \left(x-x^{\prime}, y-y^{\prime}, d+d^{\prime}(\bmod 4)\right), & \text { if } d=2, \\ \left(x+y^{\prime}, y-x^{\prime}, d+d^{\prime}(\bmod 4)\right), & \text { if } d=3 .\end{cases}
$$

These rules are obtained by rotating the vector $\left(x^{\prime}, y^{\prime}\right)$ by the angle corresponding to $d$ and adding this vector to the vector $(x, y)$. Notice that in order to find the configuration for the word $Y Z$ we do not have to compute configurations letter by letter, if we know the configurations for the words $Y$ and $Z$. The configuration of the word $Y Z$ depends only on configurations of the words $Y$ and $Z$. Actually the condition (3.2) defines a product on the set $\mathbb{Z}^{2} \times \mathbb{Z}_{4}$. We will denote the operation of product of configurations $(x, y, d)$ and $\left(x^{\prime}, y^{\prime}, d^{\prime}\right)$ by $*:\left(x^{\prime \prime}, y^{\prime \prime}, d^{\prime \prime}\right)=$ $(x, y, d) *\left(x^{\prime}, y^{\prime}, d^{\prime}\right)$. When configurations are denoted by Greek letters, we will skip $*$ for brevity. The set of configurations with operation defined by (3.2) form a group with unit element being $\varepsilon=(0,0,0)$, and the inverse element is defined as follows:

$$
(x, y, d)^{-1}= \begin{cases}(-x,-y, 2), & \text { if } d=0, \\ (-y, x, 3), & \text { if } d=1, \\ (x, y, 2), & \text { if } d=2, \\ (y,-x, 1), & \text { if } d=3 .\end{cases}
$$

We say that a configuration $\left(x^{\prime}, y^{\prime}, d^{\prime}\right)$ is a power of a configuration $(x, y, d)$, if it is obtained as a product of several copies of $(x, y, d)$. Note that configurations 
with $d=1$ and $d=3$ are elements of order 4 , configurations with $d=2$ are elements of order 2 , and $d=0$ corresponds to elements of infinite order (except for the configuration $(0,0,0))$. For example, powers of a configuration $(x, y, 1)$ are $(x, y, 1),(x-y, y+x, 2),(-y, x, 3)$ and $(0,0,0)$.

We associate to the locally catenative sequence $X_{n}$ defined by (3.1) the sequence $\left(\alpha_{n}\right)_{n>0}$ of configurations, i.e., $\alpha_{n}=\operatorname{conf} X_{n}$. The goal of this section is the following theorem:

Theorem 3.1. Let $X$ be a locally catenative sequence over a finite alphabet $\Sigma$ corresponding to the recurrence relation $X_{n}=X_{n-1} X_{n-i_{1}} \ldots X_{n-i_{k}}$ with initial values $X_{0}, \ldots, X_{q-1}$, and let $q=\max \left(i_{1}, \ldots, i_{k}\right)$. The corresponding curve, with any drawing instructions, is bounded if and only if there exists an integer $N \leq 2 q 4^{q}$, such that configurations of $X_{N}, X_{N+1}, \ldots, X_{N+q-1}$ are powers of a single finite configuration.

First we prove the following characterization for the boundedness of figures defined by a locally catenative sequence $X$ :

Lemma 3.2. Let $X$ be a locally catenative sequence over a finite alphabet $\Sigma$ corresponding to the recurrence relation $X_{n}=X_{n-1} X_{n-i_{1}} \ldots X_{n-i_{k}}$ with initial values $X_{0}, \ldots, X_{q-1}$, where $q=\max \left(i_{1}, \ldots, i_{k}\right)$. The corresponding curve, with any drawing instructions, is bounded if and only if the corresponding sequence $\alpha_{n}$ of configurations is ultimately periodic. Moreover, the configurations are ultimately powers of a single configuration of finite order.

Proof. The proof essentially consists of three parts. First we introduce auxiliary notation, then we prove the sufficiency of these conditions, and then the necessity. The sufficiency is proved straightforwardly by indicating a finite subset of $\mathbb{Z}^{2}$ and proving that the curve is contained in this subset. To prove the necessity of these conditions, we first prove ultimate periodicity of the sequence of configurations and then show that in the case of bounded curve configurations have to be of a certain form.

We will need some additional notation. Denote by $\mathrm{Sq}_{C}(t, z)$ the square with center at the point $(t, z)$ and side $2 C$, i.e., $\mathrm{Sq}_{C}(t, z)=[t-C, t+C] \times[z-$ $C, z+C]$. Let $Y, Z$ be two words with configurations $\operatorname{conf}(Y)=(x, y, d)$ and $\operatorname{conf}(Z)=\left(x^{\prime}, y^{\prime}, d^{\prime}\right)$; let pictures corresponding to these words be inside the unions of squares $\bigcup_{i=1}^{l} \mathrm{Sq}_{C}\left(t_{i}, z_{i}\right)$ and $\bigcup_{i=1}^{l^{\prime}} \mathrm{Sq}_{C}\left(t_{i}^{\prime}, z_{i}^{\prime}\right)$, respectively. Consider the picture corresponding to the word $Y Z$. One can notice from (3.2) that in the product $\left(x^{\prime \prime}, y^{\prime \prime}, d^{\prime \prime}\right)$ of configurations $(x, y, d)$ and $\left(x^{\prime}, y^{\prime}, d^{\prime}\right)$ the point $\left(x^{\prime \prime}, y^{\prime \prime}\right)$ does not depend on the direction $d^{\prime}$. When the picture corresponding to the word $Z$ is rotated and translated corresponding to the configuration $\operatorname{conf}(Y)$, the square $\mathrm{Sq}_{C}\left(t_{i}^{\prime}, z_{i}^{\prime}\right)$ moves to the point defined by the product of the configurations $(x, y, d)$ and $\left(t_{i}^{\prime}, z_{i}^{\prime}, \cdot\right)$, where $\cdot$ denotes any direction, i.e., the square $\mathrm{Sq}_{C}\left(t_{i}^{\prime}, z_{i}^{\prime}\right)$ moves to the square $\operatorname{Sq}_{C}\left(\left.\left((x, y, d) *\left(t_{i}^{\prime}, z_{i}^{\prime}, \cdot\right)\right)\right|_{\mathbb{Z}^{2}}\right)$. So the picture corresponding to the word 
$Y Z$ is inside the union of squares

$$
\bigcup_{i=1}^{l} \operatorname{Sq}_{C}\left(t_{i}, z_{i}\right) \bigcup_{i=1}^{l^{\prime}} \operatorname{Sq}_{C}\left(\left.\left((x, y, d) *\left(t_{i}^{\prime}, z_{i}^{\prime}, \cdot\right)\right)\right|_{\mathbb{Z}^{2}}\right) .
$$

This corresponds to the union of the picture corresponding to the word $Y$ and the picture corresponding to the word $Z$ rotated and translated accordingly to $\operatorname{conf}(Y)$.

First we will prove the sufficiency of the conditions, i.e., if the sequence of configurations ultimately consists of powers of some configuration of finite order, then the corresponding picture is bounded. Notice that if the sequence $\alpha_{n}$ contains $q$ successive elements equal to powers of one configuration $\alpha$ of finite order, then all further elements are powers of $\alpha$. Denote by $N$ the number from which the period in configurations starts.

There exists a constant $C \in \mathbb{Z}$ such that on the step $N+q-1$ the picture is inside the square $\mathrm{Sq}_{C}(0,0)$; on previous steps the pictures are parts of this picture, so they are also inside this square. The picture is inside the following union of squares (just because it is inside one of these squares corresponding to $i=0$ ):

$$
\bigcup_{i=0}^{3} \operatorname{Sq}_{C}\left(\left.\alpha^{i}\right|_{\mathbb{Z}^{2}}\right)
$$

Using rules (3.2) and (3.4), we obtain that product of any two powers of $\alpha$ with pictures inside this union of squares gives a power of $\alpha$ with picture inside the same union of squares. Therefore, the picture is bounded.

Now we will prove the necessity of the conditions: if the curve is bounded, then the sequence of configurations ultimately consists of periodic powers of a single configuration.

If the whole curve is bounded, then it has only a finite number of configurations in the end of iterations, denote this number by $m$. The configuration on every step is defined by previous $q$ configurations. There are at most $m^{q}$ sequences of $q$ configurations, so two identical sequences of $q$ configurations can be found at distance not greater than $m^{q}$. Therefore, the sequence of configurations is ultimately periodic with the period $T \leq m^{q}$. Denote by $N$ the length of the threshold in the ultimately periodic sequence of configurations.

Now we will prove, that in the case of bounded curve there exists a finite configuration $\alpha$, such that for $n \geq N$ all configurations $\alpha_{n}$ are powers of $\alpha$.

Denote by $\langle\beta, \gamma\rangle$ the monoid, generated by configurations $\beta$ and $\gamma$. We will prove the following claim:

Claim 1. If the curve is bounded and for $n, m \geq N$ we have $\alpha_{n}=\beta$ and $\alpha_{m}=\gamma$, then the curve goes through configurations $\langle\beta, \gamma\rangle$.

We will prove this claim by induction. Denote the period of the ultimately periodic sequence of configurations by $T$, then $\alpha_{n+T j}=\beta$ and $\alpha_{m+T j}=\gamma$ for an integer $j$. We assume that the period is not less than the order of the recurrence 
relation $T \geq q$, otherwise we multiply the period by integer large enough. Since the sequence of configurations is ultimately periodic, without loss of generality we suppose that $m, n \geq q, 0<m-n<T-q$.

We will prove that, for all $j \in \mathbb{N}$, on the iteration $m+T j+1$ the curve goes through the configurations $\left\{\prod_{i=1}^{j+1} \delta_{i} \mid \delta_{i}=\beta\right.$ or $\left.\gamma\right\}$. Actually it means that the curve passes through configurations which are obtained as product of $j+1$ multiples each of which is either $\beta$ or $\gamma$.

The curve goes through the configuration $\varepsilon$, because we started from it. On the steps $n$ and $m$ we have the configurations $\beta$ and $\gamma$, respectively. Therefore, we have the induction base for $j=0$. Suppose we have the condition on the step $m+T(j-1)+1$, i.e., the curve goes through the configurations $\left\{\prod_{i=1}^{j} \delta_{i} \mid \delta_{i}=\beta\right.$ or $\left.\gamma\right\}$. On the step $n+T j+1$ we join the curve on step $n+T j$ with configuration $\beta$ with the curve from step $n+T j-i_{1}$ translated and rotated correspondingly to $\beta$. Therefore, the curve goes through the configurations $\left\{\beta \prod_{i=1}^{j} \delta_{i} \mid \delta_{i}=\beta\right.$ or $\left.\gamma\right\}$. Symmetrically, on the step $m+T j+1$ we join the curve on step $m+T j$ with configuration $\gamma$ with the curve from step $m+T j-i_{1}$ translated and rotated corresponding to $\beta$. Therefore, the curve goes through the configurations $\left\{\gamma \prod_{i=1}^{j} \delta_{i} \mid \delta_{i}=\beta\right.$ or $\left.\gamma\right\}$. So, joining this set with the set of configurations obtained on the step $n+T j+1$, we get that the curve goes through all the configurations from the set $\left\{\prod_{i=1}^{j+1} \delta_{i} \mid \delta_{i}=\beta\right.$ or $\left.\gamma\right\}$.

So, when $n, m$ go to infinity, the curve passes through all the configurations from the semigroup $\langle\beta, \gamma\rangle$. Notice that for bounded curve this monoid has to be finite and thus all elements in it have to be of finite order.

Claim 2. The monoid $\langle\beta, \gamma\rangle$ is finite iff $\beta$ and $\gamma$ are powers of a single configuration of finite order.

The proof of this claim consists of studying five cases of pairs of configurations depending on their directions, in each case we will prove that one of these configurations is a power of another.

(1) If $\beta=(x, y, 0)$, then necessarily $\beta=\varepsilon$;

(2) If for $\beta=(x, y, d)$ and $\gamma=\left(x^{\prime}, y^{\prime}, d^{\prime}\right)$ we have $d=d^{\prime}$, then $\beta=\gamma$, i.e., the configurations with the same $d$ are equal;

(3) if $\beta=(x, y, 1)$ and $\gamma=\left(x^{\prime}, y^{\prime}, 2\right)$, then $\gamma=\beta^{2}$;

(4) if $\beta=(x, y, 3)$ and $\gamma=\left(x^{\prime}, y^{\prime}, 2\right)$, then $\gamma=\beta^{2}$;

(5) if $\beta=(x, y, 1)$ and $\gamma=\left(x^{\prime}, y^{\prime}, 3\right)$, then $\gamma=\beta^{3}$.

In each case we will find a configuration from $\langle\beta, \gamma\rangle$ which has a finite order only when one of the configurations is a power of the other.

(1) $\beta$ has finite order only in the case $x=0, y=0$;

(2) $\beta^{2} \gamma^{2}=\left(x-y-x^{\prime}+y^{\prime}, y+x-y^{\prime}-x^{\prime}, 0\right)$ has finite order only in the case $x-y-x^{\prime}+y^{\prime}=0, y+x-y^{\prime}-x^{\prime}=0$, which implies $\beta=\gamma$;

(3) $\beta^{2} \gamma=\left(x-y-x^{\prime}, y+x-y^{\prime}, 0\right)$ has finite order only in the case $x-y-x^{\prime}=0$, $y+x-y^{\prime}=0$, which means $\gamma=\beta^{2}$;

(4) $\beta^{2} \gamma=\left(x+y-x^{\prime}, y-x-y^{\prime}, 0\right)$ has finite order only in the case $x+y-x^{\prime}=0$, $y-x-y^{\prime}=0$, which means $\gamma=\beta^{2}$; 
(5) $\beta \gamma=\left(-y+y^{\prime}, x-x^{\prime}, 0\right)$ has finite order only in the case $-y+y^{\prime}=0$, $x-x^{\prime}=0$, which means $\gamma=\beta^{3}$.

Claim 2 is proved.

Claims 1 and 2 imply that starting from the step $N$ every configuration is a power of a single configuration of finite order. Thus, we proved that the sequence of configurations is ultimately periodic, moreover, the period is equal to the period in directions. Lemma 3.2 is proved.

Now we are going to establish an upper bound for the threshold of the ultimately periodic sequence of configurations in the case of bounded picture. We will need the following auxiliary lemma, which can be probably obtained in a different way using some techniques from matrix theory.

Lemma 3.3. Consider a vector recurrence relation of the form

$$
\bar{y}^{n}=A \bar{y}^{n-1},
$$

where $\bar{y}^{i}$ are vectors of length $l, A$ is a square matrix of order $l$. Let $\bar{y}^{0}$ be initial value, such that the sequence $\left(\bar{y}^{n}\right)_{n \geq 0}$ is ultimately constant. Then the length of the threshold is less than $l$.

Proof. Let $J$ be the Jordan form of $A$ via the matrix $Q$ of coordinate transformation, i.e., $A=Q J Q^{-1}$ [11]. Then the recurrence relation (3.5) can be written in the form

$$
\bar{z}^{n}=J \bar{z}^{n-1}
$$

where $\bar{z}^{n}=Q \bar{y}^{n}$. If $\bar{y}^{n}$ is ultimately constant, then $\bar{z}^{n}$ is also ultimately constant. Denote by $n_{0}$ the number from which these sequences are constant, i.e., $\bar{z}^{n}=\bar{z}^{n_{0}}$ for $n \geq n_{0}, \bar{z}^{n_{0}-1} \neq \bar{z}^{n_{0}}$. We suppose that $n_{0}>0$, otherwise there is nothing to prove. So we have that $\bar{z}^{n_{0}}=J \bar{z}^{n_{0}}, \bar{z}^{n_{0}}$ is an eigenvector corresponding to the eigenvalue $1, \bar{z}^{n_{0}}=J \bar{z}^{n_{0}-1}$.

The idea of the proof of this lemma is reconstructing the sequence $\bar{z}^{n}$ in backwards direction, starting from $n_{0}$ and using the recurrence relation (3.6). First we find out how $\bar{z}^{n_{0}-1}$ can look like, using the recurrence relation for $n=n_{0}$, then $\bar{z}^{n_{0}-2}$ and so on. To do this we need several simple observations. We split vectors $\bar{z}^{n}$ into blocks corresponding to Jordan cells of the matrix $J$ and consider each part separately. Denote by $J_{l}^{\lambda}$ the Jordan cell of order $l$ with eigenvalue $\lambda$.

Claim 1. If $J_{l}^{0} \bar{x}=\bar{y}$, then $y_{l}=0, x_{i}=y_{i-1}$ for $i=2, \ldots, l$ and $x_{1}$ can be arbitrary.

Claim 2. If $J_{l}^{1} \bar{y}=\bar{x}$, then $x_{i}=y_{i}-y_{i+1}$ for $i=1, \ldots, l-1, x_{l}=y_{l}$.

Claim 3. If $J_{l}^{\lambda} \bar{x}=\overline{0}, \lambda \neq 0$, then $x_{i}=0$ for $i=1, \ldots, l$.

We have that $\bar{z}^{n_{0}}$ is an eigenvector corresponding to the eigenvalue 1 . The equation $\bar{z}^{n_{0}}=J \bar{z}^{n_{0}}$ and Claims 1, 2 imply that in $\bar{z}^{n_{0}}$ blocks corresponding to cells with eigenvalue 1 are vectors with zeros in all coordinates of blocks except the first one which can be arbitrary. Blocks corresponding to cells with other eigenvalues are zero vectors. 
Claim 3 implies that all the values in blocks corresponding to Jordan cells with $\lambda \neq 0,1$ in vectors $\bar{z}^{0}, \ldots, \bar{z}^{n_{0}-1}$ are equal to 0 .

Now consider the behavior of values in blocks of $\bar{z}^{n}$ corresponding to the eigenvalue 1. Consider such block of size $j$ and denote it by $\bar{a}^{n}=\left(a_{1}^{n}, \ldots, a_{j}^{n}\right)$. For the vectors $\bar{a}^{n}$ we have the recurrence relation $\bar{a}^{n}=J_{l}^{1} \bar{a}^{n-1}$. Since $\bar{z}^{n_{0}}$ is an eigenvector corresponding to the eigenvalue 1 , we have $a_{2}^{n_{0}}=0, \ldots, a_{j}^{n_{0}}=0, a_{1}^{n_{0}}$ can be arbitrary. From the recurrence relation for $\bar{a}^{n_{0}}$ and Claim 2 we conclude that $\bar{a}^{n_{0}-1}=\bar{a}^{n_{0}}$. Applying the recurrence relation for $i=n_{0}-2, \ldots, 2$, we get that $\bar{a}^{i}=\bar{a}^{n_{0}}$, so the values from the blocks corresponding to Jordan cells with eigenvalue 1 are constant from the very beginning.

Now consider the values in blocks of $\bar{z}^{n}$ corresponding to the eigenvalue 0 . Consider such block of length $m$ and denote corresponding block in $\bar{z}^{n}$ by $\bar{b}^{n}=$ $\left(b_{1}^{n}, \ldots, b_{m}^{n}\right)$. For the vectors $\bar{b}^{n}$ we have the recurrence relation $\bar{b}^{n}=J_{l}^{1} \bar{b}^{n-1}$. Since $\bar{z}^{n_{0}}$ is an eigenvector corresponding to the eigenvalue 1 , we have $\bar{b}^{n_{0}}=\overline{0}$. From the recurrence relation for $\bar{b}^{n_{0}}$ and Claim 1 we conclude that $b_{2}^{n_{0}-1}=0, \ldots, b_{m}^{n_{0}}=0$, $b_{1}^{n_{0}-1}$ can be arbitrary nonzero (if it equals zero, there exist another block with nonzero eigenvalue, because $\left.\bar{z}^{n_{0}} \neq \bar{z}^{n_{0}-1}\right)$. Applying the recurrence relation for $\bar{b}^{n_{0}-i}, i=1, \ldots, m-1$, we get that $b_{2}^{n_{0}-i-1}=b_{1}^{n_{0}-i}, \ldots, b_{i+1}^{n_{0}-i-1}=b_{i}^{n_{0}-i}=b_{1}^{n_{0}-1}$, $b_{i+2}^{n_{0}-i-1}=b_{i+1}^{n_{0}-i}=0, \ldots, b_{m}^{n_{0}-i-1}=b_{m-1}^{n_{0}-i}=0, b_{1}^{n_{0}-i-1}$ can be arbitrary. For $i=$ $m-1$ we get that $b_{2}^{n_{0}-m}=b_{1}^{n_{0}-m+1}, \ldots, b_{m}^{n_{0}-m}=b_{1}^{n_{0}-1}, b_{1}^{n_{0}-m}$ can be arbitrary. The sequence of $\bar{b}^{n}$ can be written in the vector form:

$$
\bar{b}^{n_{0}-1}=\left(\begin{array}{c}
b_{1}^{n_{0}-1} \\
0 \\
\vdots \\
0
\end{array}\right), \ldots, \bar{b}^{n_{0}-i-1}=\left(\begin{array}{c}
b_{1}^{n_{0}-i-1} \\
b_{1}^{n_{0}-i} \\
b_{2}^{n_{0}-i} \\
\vdots \\
b_{m-1}^{n_{0}-i}
\end{array}\right)=\left(\begin{array}{c}
b_{1}^{n_{0}-i-1} \\
b_{1}^{n_{0}-i} \\
\vdots \\
b_{1}^{n_{0}-1} \\
0 \\
\vdots \\
0
\end{array}\right), \ldots, \bar{b}^{n_{0}-m}=\left(\begin{array}{c}
b_{1}^{n_{0}-m} \\
\\
\vdots \\
b_{1}^{n_{0}-1}
\end{array}\right) .
$$

By Claim 1 we have that $b_{m}^{n_{0}-m}=0$. But this is impossible since $b_{m}^{n_{0}-m}=b_{1}^{n_{0}-1}$, so $n_{0}<m$.

Therefore, the length of the threshold is bounded by the size of the largest Jordan cell in the matrix $A$, so it is less than $m$, and $m$ is less than $l$. Lemma 3.3 is proved.

Lemma 3.4. Let $X$ be a locally catenative sequence over a finite alphabet $\Sigma$ corresponding to the recurrence relation $X_{n}=X_{n-1} X_{n-i_{1}} \ldots X_{n-i_{k}}$ with initial values $X_{0}, \ldots, X_{q-1}$, and let $q=\max \left(i_{1}, \ldots, i_{k}\right)$. If the curve defined by turtle graph$i c s$ is bounded, then the length of the threshold in the ultimately periodic sequence $\left(\alpha_{n}\right)_{n \geq 1}$ of configurations is less than $2 q 4^{q}$.

Proof. Consider the sequence of configurations for a locally catenative sequence with bounded picture. From Lemma 3.2 it follows that the sequence of configurations is ultimately periodic; denote the period by $T$. Notice that $T \leq$ $4^{q}$. For every $n$ the values $x_{n}$ and $y_{n}$ can be found as a linear combination of 
$x_{n-1}, y_{n-1}, \ldots, x_{n-q}, y_{n-q}$; the coefficients of the linear combination depend only on $n \bmod$ T. Applying corresponding linear combinations for smaller indices, we can calculate $x_{n}$ and $y_{n}$ as a linear combination of every $q$ preceding successive values of $x_{j}, y_{j}$. Therefore, we can obtain $x_{n+1}, y_{n+1}, \ldots, x_{n+q}, y_{n+q}$ as linear combinations of $x_{n-T+1}, y_{n-T+1}, \ldots, x_{n-T+q}, y_{n-T+q}$. Denote by $\bar{v}^{i}$ the following vector of length $2 q$ :

$$
\bar{v}^{i}=\left(\begin{array}{c}
x_{K+i T+1} \\
y_{K+i T+1} \\
\vdots \\
x_{K+i T+q} \\
y_{K+i T+q}
\end{array}\right),
$$

where $K$ is the index from which the period in directions starts, i.e., $1 \leq K \leq 4^{q}$. Then

$$
\bar{v}^{i}=A \bar{v}^{i-1},
$$

where $A$ is the matrix of coefficients of linear combinations for $\bar{v}^{i}$ through $\bar{v}^{i-1}$.

If the picture is bounded, then the sequence $\left(x_{i}, y_{i}, d_{i}\right)$ is ultimately periodic, i.e., $\bar{v}_{i}$ is ultimately constant. Applying Lemma 3.3 to the sequence $\bar{v}^{i}$, we get that the threshold of this sequence is not greater than $l=2 q$. Therefore, the threshold of $\alpha_{n}$ is not greater than $2 q T$, where $T \leq 4^{q}$, so the upper bound for the threshold is $2 q 4^{q}$.

Proof of Theorem 3.1. The proof follows from Lemmas 3.2 and 3.4.

Remark. We note that the boundedness depends only on the recurrence relation and the configurations of the initial values, and does not depend on the path by which we come to these configurations, nor on the size of the alphabet, nor on the drawing rules.

Remark. Theorem 3.1 gives an algorithm to find out whether the curve corresponding to a recurrence relation with initial values is bounded or not. But the complexity of the algorithm is exponential, so we actually prove that the problem of determining boundedness is decidable.

Examples. Consider the application of Theorem 3.1 for curves defined by the Fibonacci recurrence. To check whether drawing instructions for he Fibonacci word give bounded picture, we should consider first $N=256$ configurations and check whether any two consecutive configurations are powers of one configuration (or, equivalently, one is a power of another). Consider drawing rules (or initial conditions) for the examples of curves for the Fibonacci recurrence from Section 2. For the initial values $F_{0}=0, F_{1}=01$ it is sufficient to consider first $N=256$ configurations and to notice that no two consecutive configurations are powers of a single configuration, so the curve is unbounded. For the initial values $F_{0}=01$, $F_{1}=01011111$ (or previous initial conditions with other drawing instructions described in Sect. 2) we have that $\operatorname{conf}\left(\mathrm{F}_{0}\right)=(-1,1,1), \operatorname{conf}\left(\mathrm{F}_{1}\right)=(-2,0,2)=$ $\left(\operatorname{conf}\left(\mathrm{F}_{0}\right)\right)^{2}$, so the curve is bounded. 


\section{The BInARY CASE With CODINGS}

In this section we consider boundedness of curves defined by the general case of recurrence relation with codings over the binary alphabet $\Sigma=\{0,1\}$ :

$$
X_{n}=X_{n-1} \mu_{1}\left(X_{n-i_{1}}\right) \ldots \mu_{k}\left(X_{n-i_{k}}\right)
$$

with the initial values

$$
X_{0}, \ldots, X_{q-1},
$$

where $q=\max \left(i_{1}, \ldots, i_{k}\right), \mu_{j}$-s are codings, i.e., 1-uniform morphisms [1].

General idea for the characterization of the boundedness in this case is similar to one from previous section, but in the case of a recurrence relation with codings the characterization and the proof are more complicated, and some special technical tricks are required. In the proof we use essentially the fact that the alphabet is binary, thought we believe that the result could be obtained also for larger alphabets.

In this section we will use some notation for intermediate steps. Denote by $X_{n, j}=X_{n-1} \mu_{1}\left(X_{n-i_{1}}\right) \ldots \mu_{j}\left(X_{n-i_{j}}\right), j=1, \ldots, k$. We will refer to step from $X_{n}$ to $X_{n+1}$ as $n$th iteration (or iteration $n$ ), and the step from $X_{n, j}$ to $X_{n, j+1}$ $\left(X_{n+1,1}\right.$ in the case $\left.j=k\right)$ as subiteration $(n, j)$. We will use the sequence of configurations $\delta_{m}$ with intermediate configurations corresponding to subiterations, i.e., $\delta_{n k+j}=\gamma_{n, j}=\operatorname{conf}\left(X_{n, j}\right), j=1, \ldots, k$. Note that $\alpha_{n}$ is a subsequence of the sequence $\delta_{m}: \delta_{n k+k}=\alpha_{n}$.

In the binary case four codings are possible:

$$
\begin{aligned}
& \mu=\mu^{i d}: \mu^{i d}(0)=0, \mu^{i d}(1)=1 ; \\
& \mu=\mu^{c o}: \mu^{c o}(0)=1, \mu^{c o}(1)=0 ; \\
& \mu=\mu^{0}: \mu^{0}(0)=0, \mu^{0}(1)=0 ; \\
& \mu=\mu^{1}: \mu^{1}(0)=1, \mu^{1}(1)=1 .
\end{aligned}
$$

Parallel with the sequence $X_{n}$ we consider the sequence $Y_{n}=\mu^{c o}\left(X_{n}\right)$. Notice that for the sequence $Y_{n}$ the following recurrence relation holds:

$$
Y_{n}=Y_{n-1} \lambda_{1}\left(Y_{n-i_{1}}\right) \ldots \lambda_{k}\left(Y_{n-i_{k}}\right)
$$

with the initial values

$$
Y_{0}=\mu^{c o}\left(X_{0}\right), \ldots, Y_{q-1}=\mu^{c o}\left(X_{q-1}\right)
$$

where

$$
\lambda_{j}= \begin{cases}\mu_{j}, & \text { if } \mu_{j}=\mu^{i d} \text { or } \mu_{j}=\mu^{c o} \\ \mu^{1}, & \text { if } \mu_{j}=\mu^{0} \\ \mu^{0}, & \text { if } \mu_{j}=\mu^{1}\end{cases}
$$


Lemma 4.1. Let $X$ be a locally catenative sequence over the binary alphabet $\Sigma=\{0,1\}$ defined by the recurrence relation $X_{n}=X_{n-1} \mu_{1}\left(X_{n-i_{1}}\right) \ldots \mu_{k}\left(X_{n-i_{k}}\right)$ with $\mu_{j}$ s being codings and initial values $X_{0}, \ldots, X_{q-1}$, where $q=\max \left(i_{1}, \ldots, i_{k}\right)$. If the curve defined by $X$ is bounded, then the corresponding sequence $\delta_{m}$ of configurations is ultimately periodic.

Proof. If the whole curve is bounded, then it has only a finite number of configurations in the end of iterations, denote this number by $m$. If the recurrence relation contains $\mu^{0}$ (or $\mu^{1}$ and $\mu^{c o}$ ), then $\operatorname{conf}(0)$ is of finite order (which is not greater than 4). Symmetric assertion holds for conf(1). Therefore, the sequences $\mu^{0}\left(X_{n}\right)$ and $\mu^{1}\left(X_{n}\right)$ have finite set of values defined by the length of $X_{n}$ modulo 4 . The sequence $\left|X_{n}\right| \bmod 4$ is periodic, so the sequences $\mu^{0}\left(X_{n}\right)$ and $\mu^{1}\left(X_{n}\right)$ are also periodic. Notice that we can find $X_{n}$ (and $Y_{n}$ ) from recurrence relations without using codings $\mu^{c o}$, since we can consider the sequence $X_{0}, Y_{0}, X_{1}, Y_{1}, \ldots$ and take $Y_{n-i_{j}}$ instead of $\mu^{c o}\left(X_{n-i_{j}}\right)$ (and $X_{n-i_{j}}$ instead of $\mu^{c o}\left(Y_{n-i_{j}}\right)$ ). So, the configuration on every step is defined by previous $2 q$ configurations and configurations from periodic sequences $\mu^{0}\left(X_{n}\right)$ and $\mu^{1}\left(X_{n}\right)$. Therefore, by argument similar to the general case without codings, we get that the sequence of configurations is ultimately periodic. Lemma is proved.

Remark. Notice that if the recurrent relation contains the coding $\mu^{c o}$, then the curve corresponding to $X_{n}$ is bounded if and only if the curve corresponding to $Y_{n}$ is bounded.

Denote by $L$ the length of the threshold in the ultimately periodic sequence $\delta_{m}$, i.e., pure period starts from $L$. Denote by $A$ the set of configurations in the ultimately periodic sequence $\delta_{m}$ for $m \geq L$. The set $A$ can be split into four subsets $A^{i d}, A^{c o}, A^{0}, A^{1}$, where each subset consists of configurations preceding corresponding coding in the recurrence relation. For example, if $\mu_{j}=\mu^{c o}$, then for $j \in\{2, \ldots, k\}$ we have $\gamma_{n, j-1} \in A^{c o}$, for $j=1$ we have $\gamma_{n-1, k} \in A^{c o}$, here $n \geq L / k$. Observe that these sets can intersect.

Symmetrically to the sequence $\delta_{m}$ of configurations for $X_{n}$ we define the sequence of configurations $\beta_{m}$ for the sequence $Y_{n}$, and the sets $B, B^{i d}, B^{c o}, B^{0}$, $B^{1}$.

For sets of configurations $C$ and $D$ denote by $\langle C D\rangle$ the monoid generated by products of elements from $C$ and $D$, i.e., $\langle C D\rangle=\langle\{\gamma \beta \mid \gamma \in C, \beta \in D\}\rangle$. Notice that $\langle\beta, \gamma\rangle$ is not the same as $\langle\beta \gamma\rangle$.

Theorem 4.2. Let $X$ be a locally catenative sequence over the binary alphabet $\Sigma=\{0,1\}$ defined by the recurrence relation $X_{n}=X_{n-1} \mu_{1}\left(X_{n-i_{1}}\right) \ldots \mu_{k}\left(X_{n-i_{k}}\right)$ with $\mu_{j}$-s being codings and initial values $X_{0}, \ldots, X_{q-1}$, where $q=\max \left(i_{1}, \ldots, i_{k}\right)$. The corresponding curve, with any drawing instructions, is bounded if and only if the corresponding sequence $\delta_{m}$ of configurations is ultimately periodic and there exist configurations $\varphi$ and $\psi$ of finite order such that the following conditions hold:

(1) $\left\langle A^{i d}\right\rangle=\langle\varphi\rangle$;

(2) $\left\langle B^{i d}\right\rangle=\langle\psi\rangle$;

(3) $\left\langle A^{c o} B^{c o}\right\rangle=\langle\varphi\rangle$; 
(4) $\left\langle B^{c o} A^{c o}\right\rangle=\langle\psi\rangle$;

(5) $\operatorname{conf}(0)$ is of finite order (if the recurrence relation contains the coding $\mu^{0}$ or the codings $\mu^{1}$ and $\mu^{\text {co }}$;

(6) $\operatorname{conf}(1)$ is of finite order (if the recurrence relation contains the coding $\mu^{1}$ or the codings $\mu^{0}$ and $\left.\mu^{c o}\right)$.

Remark. The conditions (1)-(6) require that the sets $A^{i d}, A^{c o}, A^{0}, A^{1}, B^{i d}$, $B^{c o}, B^{0}, B^{1}$ are non-empty; if this is not the case, conditions using empty sets are omitted in a natural way.

Proof. First we will prove, that if the curve is bounded, then the conditions (1)-(6) hold, i.e., we start with proving the necessity of conditions (1)-(6). After that we will prove the sufficiency. Actually, in this proof we develop the method from previous section for the case of recurrence relation with codings.

(1), (2) The proofs are symmetric and similar to the proof of Lemma 3.2. We give a sketch of proof of 1). Lemma 4.1 implies that the sequence $\delta_{m}$ of configurations is ultimately periodic, denote by $N$ the threshold in this ultimately periodic sequence. So, we prove the assertion analogous to to Claim 1 from the proof of Lemma 3.2: if the curve is bounded and for $n, m \geq N, \delta_{n}, \delta_{m} \in A^{i d}$ we have $\delta_{n}=\beta$ and $\delta_{m}=\gamma$, then the curve goes through configurations $\langle\beta, \gamma\rangle$. The proof is just repeating of the argument of Claim 1. Next we apply Claim 2 from the proof of Lemma 3.2 and (1) follows.

(3), (4) We will prove the necessity of both conditions simultaneously. The proof consists of two steps. First we prove that the monoid $\left\langle A^{c o} B^{c o}\right\rangle$ consists of powers of a single finite configuration. Secondly, we prove that this configuration is the same as for $\left\langle A^{i d}\right\rangle$.

So, let $M^{\prime}$ be the maximum of lengths of thresholds in the ultimately periodic sequences $\delta_{m}$ and $\beta_{m}$, and $T^{\prime}=T k$ be their common period. Without loss of generality $M^{\prime}=k M$ for some integer $M$, i.e., we use $M^{\prime}$ and $T^{\prime}$ for indices of sequences $\delta_{m}, \beta_{m}$ with subiterations. We assume that the period is not less than the order of the recurrence relation $T \geq q$, otherwise we multiply the period by integer large enough.

We will prove by induction that, for all $j \in \mathbb{N}$, the $X_{M+T(4 j+3) \text {-curve goes }}$ through the configurations $\left(A^{c o} B^{c o}\right)^{j}$, and the $Y_{M+T(4 j+3)^{-}}$-curve goes through the configurations $\left(B^{c o} A^{c o}\right)^{j}$.

These curves go through the configuration $\varepsilon$, because we started from it. On the iteration $M+T$ (first pure period) the $X_{M+T}$-curve and $Y_{M+T}$-curve pass through the configurations $A^{c o}$ and $B^{c o}$, respectively.

Now we skip one period in order to use all the configurations we obtained at previous step. During the period starting in $M+2 T$ and ending in $M+3 T$ on every subiteration $(n, l)$ corresponding to a configuration $\xi$ from $A^{c o}$ we add to the $X_{n, l^{-}}$ curve the translated and rotated $Y_{n-i_{l+1}}$-curve according to the recurrence relation. Therefore, the $X_{n, l+1}$-curve passes through configurations obtained by product of $\xi$ and configurations through which the $Y_{n-i_{l+1}}$-curve passes, i.e., $\xi B^{c o}$. Running though the period starting in $M+2 T$ and ending in $M+3 T$ and through all $\xi$ 
from $A^{c o}$, we get that the $X_{M+3 T^{-c u r v e}}$ passes through $A^{c o} B^{c o}$. Symmetrically

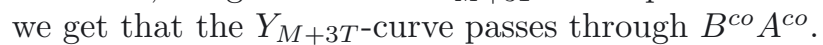

Therefore, we have the induction base for $j=1$. Suppose we have the condition for $j$.

During the period starting in $M+4 j T$ and ending in $M+(4 j+1) T$ on every subiteration $(n, l)$ corresponding to a configuration $\xi$ from $A^{\text {co }}$ we add to the $X_{n, l^{-}}$ curve the translated and rotated $Y_{n-i_{l+1}}$-curve for $i$ defined by recurrence relation. Therefore, the $X_{n, l+1}$-curve passes through configurations obtained by product of $\xi$ and configurations through which $Y_{n-i_{l+1}}$-curve passes, i.e., $\xi\left(B^{c o} A^{c o}\right)^{j}$. Running though the period starting in $M+4 j T$ and ending in $M+T(4 j+1)$ and through all $\xi$ from $A^{c o}$, we get that the $X_{M+T(4 j+1)^{-}}$-curve passes through $A^{c o}\left(B^{c o} A^{c o}\right)^{j}$.

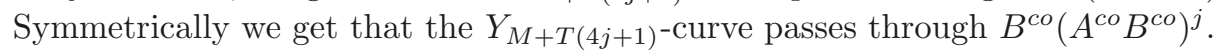

Continuing this line of reasoning, we get that during the period starting in $M+T(4 j+2)$ and ending in $M+T(4 j+3)$ the $X_{M+T(4 j+3)^{-c u r v e ~ p a s s e s ~ t h r o u g h ~ t h e ~}}$ configurations $\xi B^{c o}\left(A^{c o} B^{c o}\right)^{j}$ and thus through $A^{c o} B^{c o}\left(A^{c o} B^{c o}\right)^{j}=\left(A^{c o} B^{c o}\right)^{j+1}$. Symmetrically we get that the $Y_{M+T(4 j+3)}$-curve passes through $\left(B^{c o} A^{c o}\right)^{j+1}$.

So, when $j$ goes to infinity, the $X$-curve and $Y$-curve pass through all the configurations from the semigroups $\left\langle A^{c o} B^{c o}\right\rangle$ and $\left\langle B^{c o} A^{c o}\right\rangle$, respectively. So for boundedness of curves these monoids should be finite, hence, by what we proved in Lemma 3.2, in each of them all the elements should be powers of a single finite configuration.

It remains to prove that these generating configurations for monoids $\left\langle A^{c o} B^{c o}\right\rangle$ and $\left\langle B^{c o} A^{c o}\right\rangle$ coincide with ones from $\left\langle A^{i d}\right\rangle$ and $\left\langle B^{i d}\right\rangle$, respectively. It holds that the $X$-curve and $Y$-curve pass through the configurations from the monoids $\left\langle A^{i d}, A^{c o} B^{c o}\right\rangle$ and $\left\langle B^{i d}, B^{c o} A^{c o}\right\rangle$, correspondingly. We omit the proof of this fact, because it is similar to what we have just proved above and to Claim 1 from the proof of Lemma 3.2. The monoids $\left\langle A^{i d}, A^{c o} B^{c o}\right\rangle$ and $\left\langle B^{i d}, B^{c o} A^{c o}\right\rangle$ are finite iff $\left\langle A^{i d}\right\rangle=\left\langle A^{c o} B^{c o}\right\rangle=\langle\varphi\rangle$ and $\left\langle B^{i d}\right\rangle=\left\langle B^{c o} A^{c o}\right\rangle=\langle\psi\rangle$.

Therefore, the necessity of (3) and (4) is proved.

(5), (6) See proof of Lemma 4.1.

Now we should prove the sufficiency of conditions (1)-(6), i.e., the ultimate periodicity of the sequences of configurations and conditions (1)-(6) imply boundedness of the curve.

There exist constants $C_{1}, C_{2} \in \mathbb{Z}$ such that on the iteration $M$ the pictures $X_{M}$ and $Y_{M}$ are inside the squares $\mathrm{Sq}_{C_{1}}(0,0)$ and $\mathrm{Sq}_{C_{2}}(0,0)$, respectively. Let $C_{3}$ be an integer such that the curves corresponding to sequences from $\{0,1\}^{i}, i \leq 4 k$, lie inside the square $\mathrm{Sq}_{C_{3}}(0,0)$. This set of curves includes all curves we can obtain with codings $\mu^{0}, \mu^{1}$ in one iteration when going from $X_{n}$ to $X_{n+1}$ and from $Y_{n}$ to $Y_{n+1}$. Let $C=\max \left(C_{1}, C_{2}, C_{3}\right)$.

We will prove that the $X_{n^{-}}$-curve is inside the union of squares $\operatorname{Sq}_{C}\left(\left.\eta\right|_{\mathbb{Z}^{2}}\right)$, where $\eta$ runs over the set

$$
\begin{aligned}
& S_{X}=\langle\varphi\rangle \bigcup\langle\varphi\rangle A^{0} \operatorname{conf}(0) \bigcup\langle\varphi\rangle A^{1} \operatorname{conf}(1) \bigcup\langle\varphi\rangle A^{c o} \bigcup \\
& \bigcup\langle\varphi\rangle A^{c o} B^{0} \operatorname{conf}(0) \bigcup\langle\varphi\rangle A^{c o} B^{1} \operatorname{conf}(1) ;
\end{aligned}
$$


and the $Y_{n}$-curve is inside the union of squares $\operatorname{Sq}_{C}\left(\left.\eta\right|_{\mathbb{Z}^{2}}\right)$, where $\eta$ runs over the set

$$
\begin{aligned}
& S_{Y}=\langle\psi\rangle \bigcup\langle\psi\rangle B^{0} \operatorname{conf}(0) \bigcup\langle\psi\rangle B^{1} \operatorname{conf}(1) \bigcup\langle\psi\rangle B^{c o} \bigcup \\
& \bigcup\langle\psi\rangle B^{c o} A^{0} \operatorname{conf}(0) \bigcup\langle\psi\rangle B^{c o} A^{1} \operatorname{conf}(1) .
\end{aligned}
$$

At the iteration $M$ the pictures are inside these unions of squares (just because they are inside one of these squares). Now we will prove that if after the subiteration $(n, j)$ the pictures are covered by these sets of squares, then they will be covered with these sets after the subiteration $(n, j+1)$ (or $(n+1,1)$, if $j=k$ ). We prove it for the $X$-curve, for the $Y$-curve the proof is symmetric.

At the subiteration $(n, j)$ with $\mu_{j+1}=\mu^{i d}$ and the configuration $\gamma_{n, j}=\xi \in\langle\varphi\rangle$ we join the $X_{n, j}$-curve with the $X_{n-i_{j+1}}$-curve using the rules (3.2) and (3.4), so we obtain that the $X_{n, j+1}$-curve is inside the union of squares $\operatorname{Sq}_{C}\left(\left.\eta\right|_{\mathbb{Z}^{2}}\right)$, where $\eta$ runs over the set $\xi S_{X}$. Since $\xi \in\langle\varphi\rangle$, we have that $\xi\langle\varphi\rangle \subseteq\langle\varphi\rangle$, so $\xi S_{X} \subseteq S_{X}$.

At the subiteration $(n, j)$ with $\mu_{j+1}=\mu^{c o}$ and the configuration $\gamma_{n, j}=\chi \in A^{c o}$ we join the $X_{n, j}$-curve with the $Y_{n-i_{j+1}}$-curve using the rules (3.2) and (3.4), so we obtain that the $X_{n, j+1}$-curve is inside the union of squares $\operatorname{Sq}_{C}\left(\left.\eta\right|_{\mathbb{Z}^{2}}\right)$, where $\eta$ runs over the set $\chi S_{Y}$. First observe that

$$
\begin{aligned}
& \chi\langle\psi\rangle \subseteq A^{c o}\langle\psi\rangle=A^{c o}\left\langle B^{c o} A^{c o}\right\rangle=\left\langle A^{c o} B^{c o}\right\rangle A^{c o}=\langle\varphi\rangle A^{c o} \\
& \langle\varphi\rangle A^{c o} B^{c o}=\langle\varphi\rangle .
\end{aligned}
$$

Using these equalities, we prove separately for each subset from the union $S_{Y}$ that left multiplication by $\chi$ gives a subset of $S_{X}$ :

$$
\begin{aligned}
& \chi\langle\psi\rangle \subseteq\langle\varphi\rangle A^{c o} \subseteq S_{X} \\
& \chi\langle\psi\rangle B^{0} \operatorname{conf}(0) \subseteq\langle\varphi\rangle A^{c o} B^{0} \operatorname{conf}(0) \subseteq S_{X} \\
& \chi\langle\psi\rangle B^{1} \operatorname{conf}(1) \subseteq\langle\varphi\rangle A^{c o} B^{1} \operatorname{conf}(1) \subseteq S_{X} \\
& \chi\langle\psi\rangle B^{c o} \subseteq\langle\varphi\rangle A^{c o} B^{c o}=\langle\varphi\rangle \subseteq S_{X} \\
& \chi\langle\psi\rangle B^{c o} A^{0} \operatorname{conf}(0) \subseteq\langle\varphi\rangle A^{c o} B^{c o} A^{0} \operatorname{conf}(0)=\langle\varphi\rangle A^{0} \operatorname{conf}(0) \subseteq S_{X} \\
& \chi\langle\psi\rangle B^{c o} A^{1} \operatorname{conf}(1) \subseteq\langle\varphi\rangle A^{c o} B^{c o} A^{1} \operatorname{conf}(1)=\langle\varphi\rangle A^{1} \operatorname{conf}(1) \subseteq S_{X} .
\end{aligned}
$$

Subiterations from the sets $A^{0}, A^{1}, B^{0}$ and $B^{1}$ will not give points outside these squares because of the choice $C \geq C_{3}$. In the case $A=\emptyset$ and $A^{c o}=\emptyset$ the conditions (5), (6) and ultimate periodicity imply boundedness. In fact, in this case starting from $M$ we will draw the same curve during each period. 
Therefore, the $X$-curve and $Y$-curve stay inside these sets of squares and therefore are bounded. So, the sufficiency of the conditions (1)-(6) is proved. Theorem 4.2 is proved.

\section{Concluding Remarks AND FUture Research}

In this paper we studied curves defined by locally catenative words with drawing instructions and gave an algebraic characterization for the boundedness of such figures. We focused on catenative sequences, though modifications of our method might also work for studying curves defined by other words, e.g., Sturmian words, words used for building fractal curves. The results of this paper open a number of directions of research. It would be interesting to generalize these results for larger alphabets, to provide a bound making the characterization decidable. It would be interesting also to find an efficient characterization; it is worth noting that such characterization cannot be obtained with our method. A natural question is also to find out what kind of figures are obtained in the general case. Another direction of research might be considering how many times we come to each point, mark it on the pictures by shades, i.e., black vertices are visited often, grey ones sometimes, white ones have not been visited yet. It would be interesting to consider generalizations of all these problems in higher dimensions, where instead of "turns" rotations of the space should be considered. Besides that, the algebraic techniques developed in this paper might give new approaches to some other problems of combinatorics on words.

\section{REFERENCES}

[1] J.-P. Allouche and J. Shallit, Automatic Sequences: theory, applications, generalizations. Cambridge (2003).

[2] G. Allouche, J.-P. Allouche and J. Shallit, Kolam indiens, dessins sur le sable aux îles Vanuatu, courbe de Sierpinski et morphismes de monoïde. Ann. Inst. Fourier 56 (2006) 2115-2130.

[3] M. Barnsley, Fractals everywhere. Academic Press Professional, San Diego, USA (1998).

[4] J. Berstel and D. Perrin, Theory of codes. Academic Press (1985).

[5] J. Cassaigne, On extremal properties of the Fibonacci word. RAIRO-Theor. Inf. Appl. 42 (2008) 701-715.

[6] C. Choffrut, Iterated substitutions and locally catenative systems: a decidability result in the binary case, in Lindenmayer Systems: Impacts on theoretical computer science, computer graphics, and developmental biology. G. Rozenberg and A. Salomaa, Eds. Springer-Verlag, Berlin (1992) 49-92. Preliminary version: C. Choffrut, Iterated Substitutions and Locally Catanative Systems: A Decidability Result in the Binary Case. ICALP (1990) 490-500.

[7] C. Choffrut and J. Karhumäki, Combinatorics of words, in Handbook of Formal Languages. Springer (1997).

[8] F.M. Dekking, Recurrent sets. Adv. Math. 44 (1982) 78-104.

[9] F.M. Dekking, Replicating superfigures and endomorphisms of free groups. J. Comb. Theor. Ser. A 32 (1982) 315-320.

[10] M.M. France and J. Shallit, Wire bending. J. Comb. Theor. 50 (1989) 1-23.

[11] F.R. Gantmacher, The theory of matrices. Chelsea Publishing Company, New York (1962). 
[12] L. Kari, G. Rozenberg and A. Salomaa, L Systems, in Handbook of Formal Languages. Springer (1997).

[13] S. Kitaev, T. Mansour and P. Seebold, Generating the Peano curve and counting occurrences of some patterns. Automata, Languages and Combinatorics 9 (2004) 439-455.

[14] M. Lothaire, Algebraic combinatorics on words. Cambridge University Press (2002).

[15] M. Lothaire, Applied combinatorics on words. Cambridge University Press (2005).

[16] S. Papert, Mindstorms: children, computers, and powerful ideas. Basic Books, New York (1980).

[17] P. Prusinkiewicz and A. Lindermayer, The algorithmoic beauty of plants. Springer-Verlag, New York (1990).

[18] K. Saari, Private communication.

[19] P. Séébold, Tag-systems for the Hilbert Curve. Discr. Math. Theoret. Comput. Sci. 9 (2007) 213-226.

[20] J. Shallit, A generalization of automatic sequences. Theoret. Comput. Sci. 61 (1988) 1-16.

Communicated by C. Choffrut.

Received May 11, 2010. Accepted March 18, 2011. 